\title{
Commissioning of the ATLAS Liquid Argon Calorimeter
}

Julien LABBÉ*, on behalf of the ATLAS Liquid Argon Calorimeter group. LPSC, Université Joseph Fourier Grenoble 1, CNRS/IN2P3, Institut Polytechnique de Grenoble E-mail: labbeein2p3.fr

The Liquid Argon (LAr) calorimeter is one of the main sub-detectors of the ATLAS experiment at the CERN LHC collider (Geneva, Switzerland). It provides precision measurements of electrons, photons, jets and missing transverse energy produced in LHC pp collisions. The in situ commissioning of the LAr calorimeter has been ongoing since 2006. It has been extensively tested with frequent calibration runs, with cosmic ray muons, and with single proton beams circulating in the LHC ring. A selection of commissioning studies is presented. Their results show that the LAr calorimeter performs according to our expectations.

European Physical Society Europhysics Conference on High Energy Physics

July 16-22, 2009

Krakow, Poland

* Speaker. 


\section{The Liquid Argon Calorimeter of the ATLAS Experiment}

The ATLAS [1] liquid argon (LAr) calorimeter consists of three sampling calorimeters which all use LAr as ionisation medium : the $\mathrm{Pb} / \mathrm{LAr}$ electromagnetic (EM) calorimeter, the $\mathrm{Cu} / \mathrm{LAr}$ hadronic end-cap (HEC) and the CU-W/LAr forward calorimeter (FCal). This detector is able to cope with the high interaction rate and high radiation doses that characterise the LHC environment.

Its fine granularity features a large number of readout channels: 182468 . Among them, only ${ }^{1}$ $0.02 \%$ are permanently dead. In addition, $0.74 \%$ are unable to be read out but will be fixed the next time that the detector access is available. And $0.34 \%$ need a special calibration treatment, with a limited impact on their performance.

Each channel is individually calibrated by a charge injection system [2]. Data are regularly taken to extract the calibration constants which are used to compute the deposited energy with an optimal filtering method [3]. These calibration data have shown that the constants are very stable.

Cosmic muons [4] have been recorded in the LAr calorimeter since fall 2006 using first a dedicated top/bottom coincidence trigger from the ATLAS Tile calorimeter, and later standard ATLAS triggers like the level 1 calorimeter or track triggers. Data were also collected in September 2008 with the first LHC single beams, notably from events where the beam was dumped on a collimator upstream of ATLAS (called later beam splash events).

\section{Noise and Energy Deposit}

The noise is measured for each channel with random trigger events and has values within the design requirements [1]. The transverse missing energy has also been measured in events taken randomly. Its distribution is well described by a Gaussian noise model.

Cosmic muons recorded in the LAr calorimeter are minimum ionising particles (MIP). The distribution of deposited energies for projective muons is shown in Figure 1 (left) using two alter-
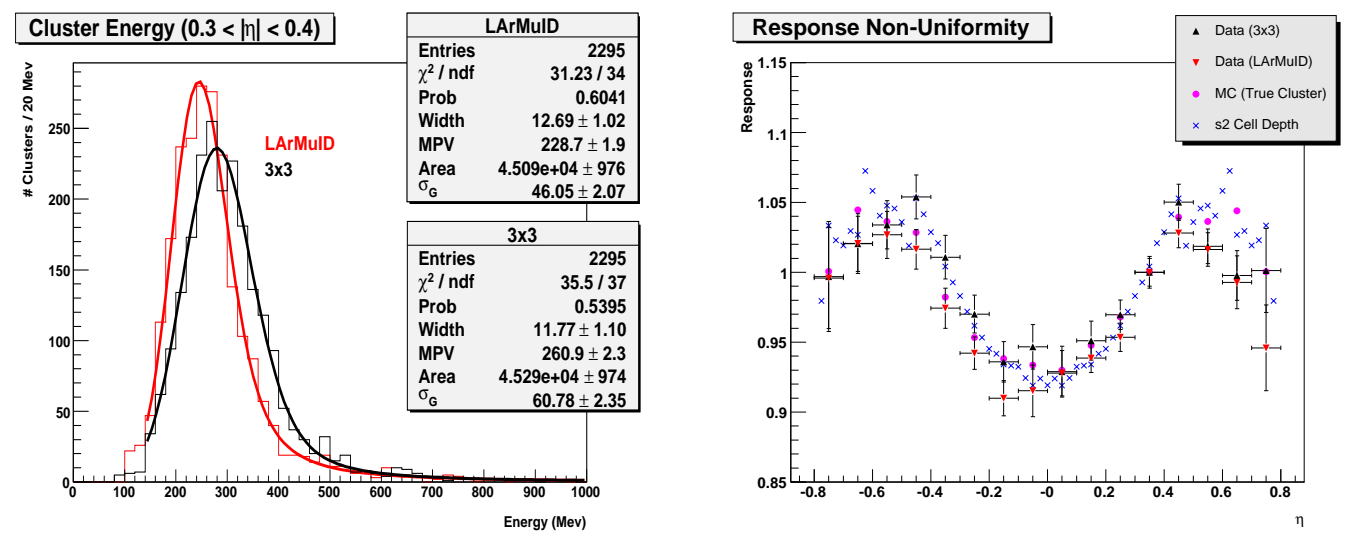

Figure 1: Left: Energy deposited in the second layer of the LAr EM calorimeter in cosmic ray events, for two different clustering methods. Right: Most probable value (MPV) of the Landau distribution as function of the pseudo-rapidity $\eta$ in data and the true cluster from the cosmic simulations. The curves have been normalised to the point at $\eta=0.35$. The cell depth in the main sampling layer, s2, is also shown.

\footnotetext{
${ }^{1}$ The status of the detector given here is that of August $18^{\text {th }}, 2009$.
} 

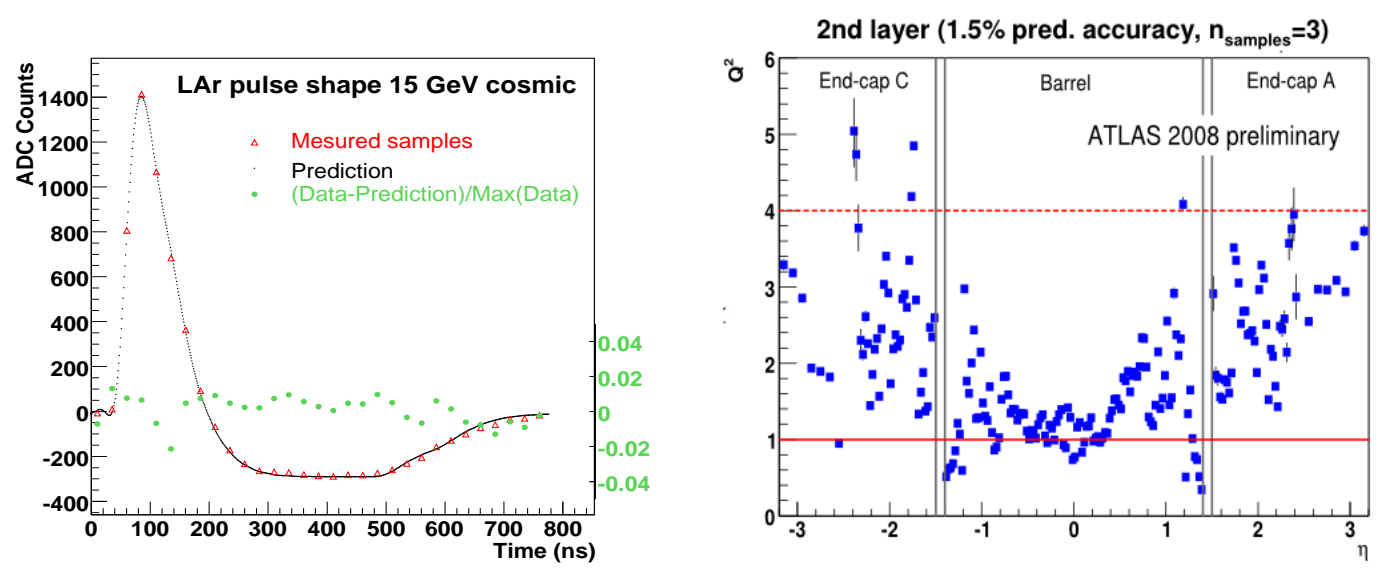

Figure 2: Left: Typical pulse for cosmic muons (data and prediction). Right: Quality factor $Q^{2}$ as function of pseudo-rapidity.

native clustering methods. The energy deposit is fitted by a Landau distribution which has been convoluted with a Gaussian to take into account electronic noise.

The most probable value (MPV) of the energy deposit distribution can be used to check the response uniformity, as shown in Figure 1 (right). The MPV value clearly tracks the cell depth, as expected for MIPs : the response uniformity agrees with simulations at the level of $2 \%$. For more details see Reference [4].

\section{Signal Reconstruction}

High energy deposits in the calorimeter readout cells are used to validate the signal shape of the calorimeter response derived from the calibration pulse. A typical pulse recorded in the EM central part is given in Figure 2 (left). The agreement with simulations is better than 2\% across the full length of the pulse.

The significant energy recorded in beam splash events, in almost every cell of the EM calorimeter, allows us to generalise this kind of study. A quality factor $Q^{2}$ has been computed:

$$
Q^{2}=\frac{1}{N_{\text {Dof }}} \cdot \sum_{i=1}^{N_{\text {samples }}} \frac{\left(A_{i}^{\text {data }}-A_{i}^{\text {pred }}\right)^{2}}{\sigma_{\text {noise }}^{2}+\sigma_{\text {pred }}^{2}}=\frac{1}{3} \cdot \sum_{i=1}^{N_{\text {samples }}} \frac{\left(A_{i}^{\text {data }}-A_{\max } \times g_{i}^{\text {phys }}\right)^{2}}{\sigma_{\text {noise }}^{2}+\left(k \cdot A_{\max }\right)^{2}},
$$

where $A_{\max }$ is the reconstructed amplitude, $g^{\text {phys }}$ the predicted physics pulse shape and $\sigma_{\text {noise }}$ the single sample noise. The prediction accuracy $k$ has been fixed to $1.5 \%$. The value of $Q^{2}$ as function of pseudo-rapidity is given in Figure 2 (right). $Q^{2} \sim N$ can be interpreted as a pulse shape prediction at the $k \sqrt{N}$ precision level. In the central region the pulse shape is predicted at $1.5 \%\left(Q^{2} \sim 1\right)$. This prediction accuracy is degraded in the lateral regions to at most $3 \%\left(Q^{2} \sim 4\right)$. These results show that the signal reconstruction is in control over the full EM calorimeter coverage.

The beam splash data contain high amplitude signals with a common time reference that are useful to perform precision timing studies. The time extracted with the optimal filtering method (corrected for the expected time of flight) has been compared with predictions. The agreement is at the level of $2 \mathrm{~ns}$. 

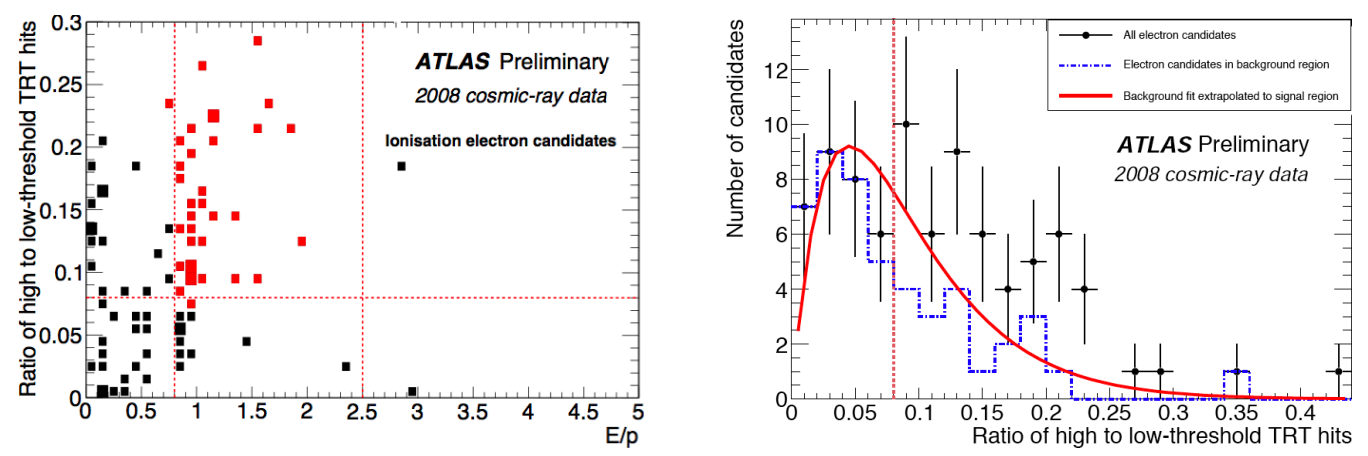

Figure 3: Left: Distribution of the events in the electron sample and definition of the signal box. Right: Distribution of the ratio of the hight to low-threshold TRT hits for the 85 electron candidates (black dots) and the 49 candidates failing the final selection (blue dashes). The red curve show the background parametrisation.

\section{Electrons from Ionisation in Cosmic Muons}

A search for electrons from ionisation has been done in the 2008 cosmic ray data. A selection has been applied on the 3.5 million events selected by the level 2 track trigger, requiring 1) a loose $\phi$ track match with EM clusters that have transverse energy above $3 \mathrm{GeV}$;2) a shower shape consistent with one from an electron ; 3) at least 25 Transition Radiation Tracker (TRT) hits. The remaining events have been split between muon bremsstrahlung candidates (1229 events with only one reconstructed track) and ionisation electron candidates ( 85 events with at least two tracks).

Two variables are used to understand the background contamination coming from bremsstrahlung events: the transition radiation measured in the TRT (inducing larger signal for relativistic particles such as electrons) and the ratio $E / p$ of the energy $E$ measured with the calorimeter to the momentum $p$ measured with the tracker ( $E / p \sim 1$ for electrons). A signal box, visible on Figure 3 (left), is defined with limits that vary with $\eta$ and $p_{T}$.

Figure 3 (right) shows the background contamination. This background is fitted with a parametrisation got from the bremsstrahlung candidate sample. A clear excess of events is observed, indicating the first observation of electrons in the ATLAS detector.

\section{Conclusion and Acknowledgements}

The ATLAS liquid argon calorimeter is now fully ready for the first LHC collisions. Commissioning results from cosmic muons and LHC single beam events are promising. The studies presented here reflect the work of the entire LAr Calorimeter group.

\section{References}

[1] G. Aad et al. [ATLAS Collaboration], JINST 3 (2008) S08003.

[2] J. Colas et al., Nucl. Instrum. Meth. A 593 (2008) 269.

[3] W. Cleland and E. Stern, Nucl. Instrum. Meth. A 338 (1994) 467.

[4] M. Cooke et. al., ATL-LARG-PUB-2007-013. 